\title{
GUIDANCE FOR THE PREVENTION OF THE COVID-19 EPIDEMIC IN LONG-TERM CARE FACILITIES: A SHORT-TERM PROSPECTIVE STUDY
}

\author{
Y. ROLLAND ${ }^{1}$, M.-H. LACOSTE ${ }^{1}$, A. DE MAULEON ${ }^{1}$, A. GHISOLFI ${ }^{1}$, P. DE SOUTO BARRETO ${ }^{1}$, \\ H. BLAIN ${ }^{2}$, H. VILLARS ${ }^{1}$
}

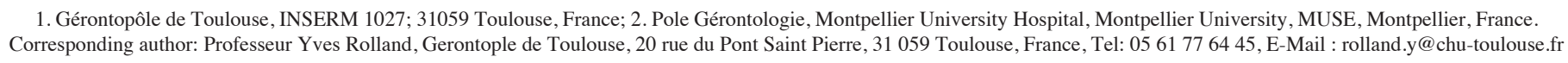

\begin{abstract}
Background: Guidance aiming at limiting the entry and spread of the COVID-19 have been widely communicated to Long-term Care Facilities (LTCFs). However, no clinical research has investigated their relevance. Objective: Our objective was to compare the guidance applied for the prevention of the COVID19 epidemic between the LTCFs having been contaminated by COVID-19 and LTCFs having not been contaminated. Methods: A questionnaire was sent and systematically accompanied by phone call to the 132 LTCFs of Haute-Garonne (Occitania region, South-West of France). The questionnaire focused on the preventive measures implemented before March 23, 2020 (first LTCFs contaminated in this area). The questionnaire focused on physician support, implementation of usual guidance (eg, masks, hydro-alcoholic solute used), training on hygiene, containment in residents' rooms and other distancing measures, use of temporary workers, compartmentalization within zones of residents and staff and a self-assessment analogic scale on the quality of the application of the preventive measures. We compared implementation of the guidance between the LTCFs with at least one case of COVID-19 among residents and/or health care professionals and LTCFs without COVID-19 case (between March 23rd and May 6th). Results: 124 LTCFs participated (93.9\%). 30 LTCFs (24.19\%) were contaminated with COVID-19. Large heterogeneity of the application of the guidance was observed. Public LTCFs (OR=0.39 (0.20-0.73), LTCFs which organized staff compartmentalization within zones $(\mathrm{OR}=0.19(0.07-0.48))$, and LTCF with a staff who self-assessed a higher quality implementation of the preventive measures $(\mathrm{OR}=0.65(0.43-0.98))$ were significantly more likely to avoid contamination by the COVID-19 outbreak. Conclusion: Our study supports the relevance of guidance to prevent the entry of COVID19 , in particular the staff compartmentalization within zones, as well as the perception of the staff regarding the quality of implementation of those measures in LTCFs.
\end{abstract}

Key words: COVID-19, long-term care facility, guidance, prevention.

\section{Introduction}

On March 11, 2020, the Ministry of Health announced, in France, the restriction of family visits and non-essential healthcare personnel in Long-Term Care (LTC) settings. This measure and active screening of anyone entering the long-term care facilities (LTCFs) for fever and symptoms of COVID19 preceded the announcement of the containment of the general population by a few days, and expressed particular concern about the spread of the COVID-19 epidemic within the LTCFs. This measure, aimed at limiting the risk of entry of the virus into the LTCFs and human-to-human contamination, was applied in most countries and was quickly accompanied by other preventive measures proposed by World Health Organization (WHO) (1), learned societies of geriatrics $(2,3)$ and various national and regional health agencies.

The broad lines of the guidance disseminated in European countries or in the United States (CDC) (4) are based on past clinical experiences and common sense in the event of an easily inter-human transmissible viral epidemic more than on scientific evidence. Such guidance has the objectives of preventing the entry of the virus in the establishment, identifying the cases early, and limiting the spread of the virus in the LTCFs. Despite this, COVID-19 preparedness in LTC was questioned (5). Indeed, these guidance were irregularly applied during the first weeks of the epidemic for several reasons: inadequate or lack of personal's protective equipment (such as masks or hydro-alcoholic gel), a shortage of rt-PCR testing kits, insufficient space to organize compartmentalization within zones, a shortage in personnel, the lack of a proper understanding/training of the vital risks for residents, and the importance for caregivers to strictly follow the barrier measures. Some guidance, such as confinement in a bedroom or the cessation of group activities, despite their interest in a public health point of view, have also raised ethical questions among nursing home staff, but also the public opinion, given their potential deleterious effects in frail subjects, especially those with cognitive impairment, behavioral issues or affective symptoms (6). This situation has led, at least at the beginning of the epidemic, to heterogeneous practices in the LTCFs and a delay in the widespread implementation of the guidance (5).

In France, 14, 178 of the 29, 319 COVID deaths (48.35\%) by June 10th 2020 (7) occurred among LTCFs residents. To our knowledge, no study to date has evaluated the differences between LTCFs with confirmed cases of COVID-19 and LTCFs without cases in terms of the effective implementation of the guidance to prevent COVID-19 contamination. Our objective was to compare the application of the guidance for 


\section{THE JOURNAL OF NUTRITION, HEALTH \& AGING}

the prevention of the COVID-19 epidemic between the LTCFs having been contaminated by at least one case of COVID-19 among residents and/or LTCFs staff and LTCFs with no cases of COVID between March 23rd and May 6th, in the year 2020.

\section{Methods}

\section{Participants}

The epidemic risk being variable over time and from one region to another, and because the guidance have evolved over time in short-term periods, our study was limited to all the LTCFs of the Haute-Garonne department (Occitania region), South-Western France, between March 23rd and May 6th, 2020. We sent a questionnaire to LTCFs directors/medical staff by email on March 30th, to all the 132 LTCFs registered in the Haute-Garonne department. After sending the questionnaire, we systematically performed a phone call (period of 6 to 19 May 2020) to the LTCFs either coordinating nurse or coordinating doctor in order to explain the questions and guide them on how to complete the questionnaire online. The questionnaire focused on the preventive measures implemented in the LTCF before March 23rd, 2020. This date refers to the first confirmed case of COVID-19 in LTCFs in the department of Haute Garonne. At that period, none of the other LTCFs had confirmed cases of COVID-19. The questionnaire therefore relates to practices at the time that preceded the arrival of the epidemic wave. This date was also chosen because it was, on the basis of our field knowledge (7), an important motivational element for local LTCFs to implement more strictly all the guidance for LTCFs. This event was indeed widely diffused in the media in the Haute Garonne department, in the official website of the association of LTCFs coordinating doctors and allowed a temporal reference of a major local event helping LTCFs staff to complete the questionnaire accurately.

\section{Variables of interest}

The questionnaire included the status of the LTC (public, private for-profit, private non-profit) and the presence of a coordinating physician (yes/no). The implementation of the guidance were listed (each recommendation was a dichotomy: yes/no) regarding: the systematic wearing of masks by the healthcare professional; access to effective masks (surgical and/or FFP2); satisfactory supply of masks; satisfactory supply of hydro-alcoholic solute; access to specific internal or external training on hygiene measures throughout the year; the use of containment in residents' rooms; during meals, separating residents to each other, respecting a distance of at least one meter (in the dining room with distance, in small groups, in bedroom); cessation of group activities for residents (with distance, in small groups); the use of interim jobs; staff compartmentalization within zones (organization of the work so that the team works in small groups in one area of the LTCF with no physical connection with the other members of the team); resident compartmentalization within zones (organization of the LTCF so that the resident live in small groups in one area of the LTCF with no possible physical connection with the other residents); specific dressing procedure at the entrance (complete daily routine dress change at the entrance and exit of the LTCF for anyone entering the LTCF). In order to judge the global prevention effort made by the healthcare team, the questionnaire ended with a selfassessment scale: To what extent do you think the nursing home staff has satisfactorily applied all the recommended «barrier» measures? (rated from 0 , not at all, to 10 , perfectly). At the end of the phone call, the coordinating nurse or coordinating doctor was asked to give a global judgment of the follow-up of the guidance by the all nursing home staff.

\section{Outcome measure}

The coordinating nurse or doctor should specify whether, in the LTCFs, one or more residents and/or one or more caregivers were infected with COVID-19 (yes/no). The cases had to be confirmed by a positive rt-PCR test. Unity of measurement was the LTCF.

\section{Ethics}

This study is a survey of the leaders of the LTCFs and no data relating to residents was collected. The approval of the ethics committee was not required.

\section{Statistical Analysis}

Qualitative variables are described as numbers and percentage [n (\%)] and quantitative variables as mean and standard deviation (SD). Bivariate analysis used the chi-square test (Fisher's exact test if applicable) and the Student's ttest for independent samples, as appropriate. In order to consider the confounding factors, a logistic regression was realized. Multicollinearity was assessed. The initial model was composed of variables found associated with a LTCFs infected by COVID-19 to a threshold of 0.20 in bivariate analysis. Then, a reduced model was performed, using a step by step backward regression. Analysis has been duplicated separately for private and public LTCFs.

\section{Results}

Among the 132 LTCFs in the region, 124 (93.9\%) agreed to participate and 8 refused. In $30 \mathrm{LTCFs}$, confirmed cases of COVID-19 (24.2\%) were found among: one or more residents ( $\mathrm{n}=6$ LTCFs), one or more caregivers $(\mathrm{n}=17)$, or both $(\mathrm{n}=$ 7). Seven LTCFs (5.6\%) had confirmed cases for two or more residents. Table 1 shows the implementation of guidance in the LTCFs with confirmed COVID cases vs. those without confirmed cases. In bivariate analyses, LTCFs ownership, staff compartmentalization within zones, use of professional interim, and organization of the meals with $\geq 1$-meter distance separating residents to each other were significantly associated with infection of COVID-19 in the LTCFs. The 
Table 1

Characteristics of LTC settings and implementation ( $\mathrm{n}, \%$ yes) of preventive measures of COVID-19 epidemic in the LTC settings with or without case of COVID infection $(n=124)$

\begin{tabular}{|c|c|c|c|}
\hline & $\begin{array}{l}\text { LTC settings without COVID case } \\
\qquad(\mathrm{n}=94)\end{array}$ & $\begin{array}{l}\text { LTC settings with at least one } \\
\text { COVID case }(n=30)\end{array}$ & $\mathbf{p}$ \\
\hline \multicolumn{4}{|l|}{ Administrative status } \\
\hline - Private for profit & $34(36.2 \%)$ & $20(66.7 \%)$ & \\
\hline - Private non for-profit & $28(29.9 \%)$ & $7(23.3 \%)$ & 0.04 \\
\hline - Public & $32(33.9 \%)$ & $3(10.0 \%)$ & \\
\hline Coordinating doctor & $86(91.5 \%)$ & $28(93.3 \%)$ & 0.55 \\
\hline Staff compartmentalization within zones & $65(69.2 \%)$ & $9(30.0 \%)$ & $<0.01$ \\
\hline Resident compartmentalization within zones & $17(18.1 \%)$ & $4(13.3 \%)$ & 0.55 \\
\hline Use of professional interim & $44(46.8 \%)$ & $21(70.0 \%)$ & 0.03 \\
\hline Specific dressing procedure at the entrance & $14(14.9 \%)$ & $2(6.7 \%)$ & 0.35 \\
\hline Wearing a mask & $75(79.8 \%)$ & $24(80.0 \%)$ & 0.98 \\
\hline Satisfactory supply of masks of masks & $42(44.7 \%)$ & $17(56.7 \%)$ & 0.51 \\
\hline Access to effective masks (surgical and / or FFP2) & $79(84.0 \%)$ & $22(73.3 \%)$ & 0.19 \\
\hline Satisfactory supply of hydro-alcoholic solute & $75(79.8 \%)$ & $26(86.7 \%)$ & 0.77 \\
\hline \multicolumn{4}{|l|}{ Access to specific training on hygiene measures } \\
\hline - internal training & $52(55.3 \%)$ & $12(40.0 \%)$ & 0.28 \\
\hline - external training & $37(39.4 \%)$ & $15(50.0 \%)$ & \\
\hline Containment in residents' rooms & $69(74.2 \%)$ & $23(76.7 \%)$ & 0.79 \\
\hline \multicolumn{4}{|l|}{ Organization of the meals } \\
\hline - no specific organization & $14(14.9 \%)$ & $4(13.3 \%)$ & \\
\hline - in the dining room with distance & $38(40.4 \%)$ & $21(70.0 \%)$ & 0.02 \\
\hline - in small groups & $1(1.1 \%)$ & $0(0.0 \%)$ & \\
\hline - in bedroom & $41(43.6 \%)$ & $5(16.67 \%)$ & \\
\hline \multicolumn{4}{|l|}{ Group activities } \\
\hline - stopped & $55(58.5 \%)$ & $20(66.7 \%)$ & \\
\hline - with distancing, & $9(9.6 \%)$ & $4(13.3 \%)$ & 0.45 \\
\hline - in small groups & $30(31.9 \%)$ & $6(20.0 \%)$ & \\
\hline $\begin{array}{l}\text { Self-assessment scale of the quality of the «barrier» measures }(0, \text { not } \\
\text { at all to } 10 \text {, perfectly); mean (standard deviation) }\end{array}$ & $8.0(1.2)$ & $7.5(1.4)$ & 0.10 \\
\hline
\end{tabular}

table 2 shows the correlates of having $\geq$ one confirmed case of COVID-19. In the fully adjusted model, as well as in the reduced model, public LTCFs, LTCFs which organized staff compartmentalization within zones and a higher perception of the LTCFs staff regarding the quality of the implementation of «barrier» measures were significantly associated with a lower likelihood of having a confirmed case of COVID-19. Staff compartmentalization within areas remains the only variable associated with the absence of COVID-19 infection in the analysis conducted separately in private (supplementary table 1) and public LTCFs (supplementary table 2).

\section{Discussion}

This study showed that LTCFs which organized staff compartmentalization within zones had a lower likelihood of having a confirmed COVID-19 case between March 23rd and May 6th 2020, among 124 LTCFs in South-Western France. This investigation also evidenced a high heterogeneity across LTCFs regarding the effective implementation of the guidance to prevent COVID-19 infection and dissemination.

Although epidemic experiences in LTCFs showed that COVID-19 can enter the LTCF even when all the guidance are implemented (3), our study reinforces the idea that preventive measures are associated with lower odds of confirmed COVID19 infection in LTCFs. In the context of a lack of resources in 
Table 2

Association of COVID-19 infection in the LTC setting and the preventive guidance implemented in the LTC settings (logistic regression) $(\mathrm{n}=124)$

\begin{tabular}{|c|c|c|c|c|}
\hline & \multicolumn{2}{|c|}{ Full model } & \multicolumn{2}{|c|}{ Reduced Model } \\
\hline & Odds Ratio (IC95\%) & $\mathbf{p}$ & Odds Ratio (IC95\%) & $\mathbf{p}$ \\
\hline Administrative status (public vs private) & $0.32(0.15-0.67)$ & 0.002 & $0.39(0.20-0.73)$ & 0.003 \\
\hline Coordinating doctor & $0.18(0.02-1.85)$ & 0.15 & - & - \\
\hline Staff compartmentalization within zones & $0.17(0.04-0.67)$ & 0.01 & $0.19(0.07-0.48)$ & 0.001 \\
\hline Resident compartmentalization within zones & $3.01(0.51-18.51)$ & 0.22 & - & - \\
\hline Use of professional interim & $1.91(0.62-5.93)$ & 0.26 & - & - \\
\hline Specific dressing procedure at the entrance & $0.81(0.10-6.34)$ & 0.84 & - & - \\
\hline Wearing a mask & $1.7(0.26-11.00)$ & 0.57 & - & - \\
\hline Satisfactory supply of masks of masks & $1.43(0.55-3.72)$ & 0.46 & - & - \\
\hline Access to effective masks & $0.54(0.14-2.10)$ & 0.37 & - & - \\
\hline Satisfactory supply of hydro-alcoholic solute & $2.10(0.61-7.24)$ & 0.24 & - & - \\
\hline Access to specific training on hygiene measures & $0.71(0.28-1.79)$ & 0.47 & - & - \\
\hline Containment in residents' rooms & $1.67(0.49-5.76)$ & 0.41 & - & - \\
\hline Organizing of the meals & $0.63(0.34-1.15)$ & 0.13 & - & - \\
\hline Group activities & $0.89(0.41-1.91)$ & 0.77 & - & - \\
\hline Self-assessment scale of the quality of the «barrier» measures & $0.55(0.33-0.93)$ & 0.03 & $0.65(0.43-0.98)$ & 0.04 \\
\hline
\end{tabular}

terms of healthcare professionals and health supplies our study is also of practical interest for guiding resources allocation.

The compartmentalization of health care professional within the limit of restricted zones is a compelling measure for LTCFs and potentially for any setting for institutionalized older adults. Its objective is to limit the risk of dissemination of the virus in the LTCFs by enhancing traffic control bundling. This measure was applied by $69 \%$ of the LTCFs without cases of COVID-19, against $30 \%$ of the LTCFs infected. The effectiveness of this measure reinforces the idea that the danger of spreading the virus comes primarily from health care professionals. Compartmentalization in patients and health care professional areas is a strategy widely implemented in hospitals (hospital team and unit dedicated to COVID patients) and highly recommended for LTCFs, having been recommended, for example for Taiwanese LTCFs, institutions that have been very little impacted by the COVID-19 outbreak (8). The compartmentalized or architectural organization of places of care is a very old strategy to fight against the spread of infections. Its importance has been previously illustrated, in the context of the COVID-19, by the experience reported in an institution in King County, Washington (9).

All guidance has been widely communicated to all institutions in France, but the quality of their implementation in practice may be a determining factor. The rating by the LTCF staff regarding the quality of the implementation of the «barrier» measures possibly reflects a mode of governance, leadership and a strong willingness in the capacity of the institution to implement all possible means to prevent the epidemic and avoid the entry and spread of the virus. Although the rating by an outside observer would have been a more objective assessment of the quality of implementation of anti-COVID-19 measures, our subjective rating suggests that feelings of self-confidence/self-mastery in the institution as a whole (eg, healthcare staff, structures in the facility) may be a determinant to prevent infections in LTCFs. This variable is also open to criticism because we cannot exclude a reversal causality.

Our study suggests that public establishments have been less exposed to the COVID-19 epidemic. During the period studied, rt-PCR tests were not readily available, and the recommendation of the Regional Health Agency (RHA) in the French Haute-Garonne department (area in which this study took place) was to screen for COVID-19 only in residents or LTCFs staff who were symptomatic for COVID-19. The improvement in the availability of rt-PCR tests has secondarily enabled (after May 6th, 2020) to widen the screening strategy of the RHA by carrying out screening tests to all LTCFs healthcare professionals and residents when a first case was detected in the institution. However, the private for-profit groups of LTCFs, positioned throughout France and in Europe, have been able to implement this strategy earlier, including systematic screening in the absence of incident cases in some institutions. It is now well-known that a substantial proportion of residents or caregivers can have COVID-19 before symptoms develop and expert groups now argue that LTCFs should use rt-PCR frequently and systematically when test kits are available (10). In our cohort of 124 LTCFs, 30 of private for 


\section{GUIDANCE FOR THE PREVENTION OF THE COVID-19 EPIDEMIC IN LONG-TERM CARE FACILITIES}

profit LTCFs (55.6\%) screened systematically with rt-PCR for COVID-19 against 6 in the private none for profit (17.14\%) and $9(25.7 \%)$ in public establishments $(\mathrm{p}=0.005)$. Our hypothesis is therefore that this wide screening modality led, during the period of interest, to the identification of a higher number of cases of COVID-19 in private institutions. Analyzes performed separately in private and public LTCFs (supplementary tables) do not suggest that private nursing homes have had less access to protective equipment than public structures as suggested in Spain (11). Staff compartmentalization within areas remains the main factor associated with COVID-19 infection in both public and private nursing homes.

Our study is limited by relatively small numbers of LTCFs. However, the epidemic as well as the guidance disseminated having been progressive, the unity of place and time of our survey conducted with all the LTCFs of a geographic area allows a more reliable interpretation of the data. In addition, the collection of data during a phone call with the clinical leader of the LTCFs by a unique research investigator made it possible to achieve exhaustive and homogenous data recording. Moreover, in the context of the outbreak, no randomized controlled trial can ethically be performed to assess preventive measures against COVID-19, and we believe evidence can only come from observational data at the moment. Finally, this is, to our knowledge, the first study assessing the relevance of the guidance to prevent the COVID-19 epidemic in LTCFs.

To conclude, the epidemic prevention strategy remains the main treatment for people living in LTCFs and this population has shown to have the most notable burden of COVID-19 cases. Our study encourages health care professionals to maintain strategies to prevent the entry and spread of the virus in LTCFs. The quality of the barrier measures applied and the fight against the spread of the virus by healthcare workers are confirmed to be important factors limiting the COVID-19 epidemic in LTCFs.

Conflict of interest: The authors report no conflict of interest.

\section{References}

1. World Health Organisation. Infection Prevention and Control guidance for Long-Term Care Facilities in the context of COVID-19. Geneva, World Health Organisation, 2020. https://apps.who.int/iris/bitstream/handle/10665/331508/WHO-2019-nCoVIPC_long_term_care-2020.1-eng.pdf (accessed 31 May 2020)

2. British Geriatrics Society. Managing the COVID-19 pandemic in care homes London, British Geriatrics Society, 2020. https://www.bgs.org.uk/resources/covid-19 managing-the-covid-19-pandemic-in-care-homes (accessed 31 May 2020)

3. American Geriatrics Society. American Geriatrics Society Policy Brief: COVID-19 and Nursing Homes. J Am Geriatr Soc. 2020;68(5):908-911. doi:10.1111/jgs.16477

4. Centers for Disease Control. Preparing for COVID-19: LTCFs, Nursing Homes Atlanta, Centers for Disease Control, 2020. https://www.cdc.gov/coronavirus/2019 ncov/healthcare-facilities/prevent-spread-inlong-term-care-facilities.html (accessed 31 May 2020)

5. Quigley DD, Dick A, Agarwal M, Jones KM, Mody L, Stone PW. COVID-19 Preparedness in Nursing Homes in the Midst of the Pandemic [published online ahead of print, 2020 Apr 28]. J Am Geriatr Soc. 2020;10.1111/jgs.16520. doi:10.1111/ jgs. 16520

6. Wang H, Li T, Barbarino P, et al. Dementia care during COVID-19. Lancet 2020;395(10231):1190-1191. doi:10.1016/S0140-6736(20)30755-8

7. https://www.santepubliquefrance.fr (accessed 11 June 2020)

8. Rolland Y, Benetos A, Villars H, Braun H, Blain H. Editorial: A COVID-19 Support Platform for Long Term Care Facilities. J Nutr Health Aging. 2020;24(5):461-462. doi:10.1007/s12603-020-1364-x

9. Yen MY, Schwartz J, King CC, Lee CM, Hsueh PR; Society of Taiwan Long-term Care Infection Prevention and Control. Guidance for protecting against and mitigating the COVID-19 pandemic in long-term care facilities [published online ahead of print, 2020 Apr 10]. J Microbiol Immunol Infect. 2020;S1684-1182(20)30097-9. doi:10.1016/j.jmii.2020.04.003

10. Arons MM, Hatfield KM, Reddy SC, et al. Presymptomatic SARS-CoV-2 Infections and Transmission in a Skilled Nursing Facility. N Engl J Med. 2020;382(22):2081 2090. doi:10.1056/NEJMoa2008457

11. Wasserman M, Ouslander JG, Lam A, Wolk AG, Morley JE, Von Preyss-Friedman S, Marco, N, Nazir A, Haimowiz D, Bessey F. Diagnostic testing for SARS Coronavirus-2 in the nursing home facility: Guidance of a Delphi panel of long-term care clinicians. J Nutr Health Aging. 2020;24

12. Rada AG. Covid-19: the precarious position of Spain's nursing homes [published correction appears in BMJ. 2020 Apr 21;369:m1586]. BMJ. 2020;369:m1554. Published 2020 Apr 20. doi:10.1136/bmj.m1554 